\title{
SPHERICAL POLYNOMIALS AND THE PERIODS \\ OF A CERTAIN MODULAR FORM
}

BY

DAVID KRAMER

\begin{abstract}
The space of cusp forms on $\mathrm{SL}_{2}(\mathbf{Z})$ of weight $2 k$ is spanned by certain modular forms with rational periods.
\end{abstract}

Kohnen and Zagier [5] have investigated those modular forms on $\mathrm{SL}_{2}(\mathbf{Z})$ whose periods (in the sense of Eichler-Shimura) are rational. As an example they consider the function

$$
f_{k, D}(z)=C \sum_{(a, b, c)} \frac{1}{\left(a z^{2}+b z+c\right)^{k}}
$$

where $D$ is a positive integer, $k$ an integer greater than one, $C$ a constant, and the summation over all triples of integers $(a, b, c)$ with $b^{2}-4 a c=D$. This function was introduced by Zagier in [13], where it was shown to be a cusp form of weight $2 k$ on $\mathrm{SL}_{2}(\mathbf{Z})$, and was later shown by Kohnen [4] to be the $D$ th Fourier coefficient of the holomorphic kernel function for the Shimura-Shintani correspondence between modular forms of half-integral and integral weight. In [6] it is shown that the $f_{k, D}$ span $S_{2 k}$, the space of cusp forms on $\mathrm{SL}_{2}(Z)$ of weight $2 k$, if and only if $L(f, k)$, the $L$-series associated to the modular form $f$, does not vanish at $s=k$, the center of the critical strip, for every Hecke eigenform $f$. It is at present only a conjecture, supported by some numerical evidence, that the $f_{k, D}$ span $S_{2 k}$ for all even $k$ (for $k$ odd, $\left.f_{k, D}=0\right)$. We study the related functions $f_{k, D, A}(z)$ where now the summation is restricted to quadratic forms $(a, b, c)$ belonging to the $\mathrm{SL}_{2}(\mathbf{Z})$ equivalence class $A$. We prove that certain finite sets of the $f_{k, D, A}$ span $S_{2 k}$. The method of proof relies on the special structure of certain ideal classes and does not indicate how the conjecture that the $f_{k, D}$ span $S_{2 k}$ might be settled.

A similar result for modular forms on an arbitrary discrete cocompact subgroup of $\mathrm{SL}_{2}(\mathbf{R})$ has been obtained by Svetlana Katok [3].

Let $\mathscr{A}$ be the set of all narrow equivalence classes of primitive binary quadratic forms of positive discriminant. Throughout $k$ will denote a positive integer, and we set $w=2 k-2$. Let $A$ be an $\mathrm{SL}_{2}(\mathbf{Z})$ equivalence class of quadratic forms. We define the following classes associated to $A$ :

$$
\theta A=\{(-c,-b,-a) \mid(a, b, c) \in A\}, \quad A^{\prime}=\{(a,-b, c) \mid(a, b, c) \in A\} .
$$

Received by the editors May 1, 1985.

1980 Mathematics Subject Classification (1985 Revision). Primary 11F11, 11F12; Secondary 11C08. 
Let $P_{k}$ denote the $\mathbf{C}$-vector space of homogeneous polynomials in two variables of degree $w$. For an equivalence class $A \in \mathscr{A}$ of forms of discriminant $D$, let

$$
P_{k, D, A}(x, y)=\sum_{Q \in A} Q(x, y)^{k-1}
$$

with the summation over the reduced forms of $A$ (a binary quadratic form $Q(x, y)=a x^{2}+b x y+c y^{2}$ is reduced if $\left.a>0, c>0, b>a+c\right)$, and then set

$$
P_{k, D, A}^{ \pm}=P_{k, D, A} \pm P_{k, D, \theta A} \text {. }
$$

We shall study the span in $P_{k}$ of $P_{k, D, A}^{ \pm}, A \in \mathscr{A}$, and prove a theorem about the space of modular forms on $\mathrm{SL}_{2}(\mathbf{Z})$ of weight $2 k$.

Definition 1. A polynomial $\pi(a, b, c)$ is called spherical with respect to $b^{2}-4 a c$ if it satisfies

$$
\left(\frac{\partial^{2}}{\partial b^{2}}-\frac{\partial^{2}}{\partial a \partial c}\right) \pi(a, b, c)=0 .
$$

Proposition 1. Let $d_{n, k}(a, b, c)$ be defined by

$$
\sum_{n=0}^{2 k-2} d_{n, k}(a, b, c) x^{n} y^{2 k-2-n}=\left(a x^{2}+b x y+c y^{2}\right)^{k-1} \text {. }
$$

The set $\left\{d_{n, k}(a, b, c), 0 \leqslant n \leqslant w\right\}$ is a basis for the space of homogeneous spherical polynomials of degree $k-1$.

For a proof see [8, the corollary to Theorem 18].

THEOREM 1. Let $\mathscr{W}_{k}^{ \pm}$be the subspaces of $P_{k}$ spanned by the collections $\left\{P_{k, D, A}^{ \pm}+\right.$ $\left.P_{k, D, A^{\prime}}^{ \pm}\right\}, A \in \mathscr{A}$.

I. Then the dimensions of $\mathscr{W}_{k}^{ \pm}$are equal to $\eta_{k}^{ \pm}$, where

$$
\eta_{k}^{+}= \begin{cases}k / 2+1, & k \text { even } \\ (k+1) / 2, & k \text { odd }\end{cases}
$$

and

$$
\eta_{k}^{-}= \begin{cases}k / 2, & k \text { even } \\ (k+1) / 2, & k \text { odd }\end{cases}
$$

II. There is an infinite subset of the set $\mathscr{A}$ of equivalence classes of forms, which we shall construct explicitly, that has the following property: any $2 k$ classes in this set have corresponding $P_{k, D, A}^{ \pm}+P_{k, D, A^{\prime}}^{ \pm}$which span $\mathscr{W}_{k}^{ \pm}$. Moreover, given any positive discriminant $D$, we can choose these classes such that each has discriminant $f^{2} D$ for some integer $f$; i.e., all of the equivalance classes are associated to module classes in the same real quadratic field.

Note. We consider $P_{k, D, A}^{ \pm}+P_{k, D, A^{\prime}}^{ \pm}$rather than $P_{k, D, A}^{ \pm}$alone, because in the course of the proof we need to assume that our summation includes the form 
$(c, b, a)$ whenever it contains $(a, b, c)$. Let

$$
\sum_{A}^{ \pm} \quad \text { mean } \sum_{A}+\sum_{A^{\prime}} \pm \sum_{\theta A} \pm \sum_{\theta A^{\prime}}
$$

where the summation is over the reduced forms in the given class. Since the coefficients of $Q(x, y)^{k-1}$ are the $d_{n, k}(a, b, c)$, it follows from Proposition 1 that to establish the dimension of $\mathscr{W}_{k}^{ \pm}$it suffices to find $w+1-\eta_{k}^{ \pm}$Q-linearly independent linear combinations of the $d_{n, k}(a, b, c)$, for each of which $\sum_{\frac{ \pm}{A}}=0$ for all $A \in \mathscr{A}$, and $\eta_{k}^{ \pm}$Q-linearly independent linear combinations of the $d_{n, k}(a, b, c)$, of which no nontrivial linear combination sums $\left(\Sigma_{A}^{ \pm}\right)$to zero for all cycles $A \in \mathscr{A}$.

We first establish the requisite number of relations. Let

$$
\phi_{n, k}(a, b, c)=d_{n, k}(a, b, c)-d_{w-n, k}(a, b, c), \quad 0 \leqslant n \leqslant w,
$$

and

$$
\begin{array}{r}
\psi_{n, k}^{ \pm}(a, b, c)=d_{n, k}(a, b-2 a, a-b+c)-d_{n, k}(c, 2 c-b, a-b+c) \\
\begin{aligned}
\mp(-1)^{k}\left[d_{n, k}(a-b+c, b-2 a, a)-d_{n, k}(c-b+a, 2 c-b, c)\right], \\
0 \leqslant n \leqslant w .
\end{aligned}
\end{array}
$$

We assert that for any $A \in \mathscr{A}$

(i) $0=\sum_{A}^{ \pm} \phi_{n, k}(a, b, c)$,

(ii) $0=\sum_{A}^{ \pm} \psi_{n, k}^{ \pm}(a, b, c)$.

That (i) is true follows immediately from the facts that $d_{w-n, k}(a, b, c)=d_{n, k}(c, b, a)$ and $(a, b, c) \in A \Leftrightarrow(c, b, a) \in A^{\prime}$. To establish (ii), let $R_{n, k}^{ \pm}(a, b, c)=d_{n, k}(a, b, c)$ $\mp(-1)^{k} d_{n, k}(c, b, a)$. Then $R_{n, k}^{ \pm}(a, b, c)= \pm R_{n, k}^{ \pm}(-c,-b,-a)$, and the result follows from [7, Proposition $3^{\prime}$ ]. From among the polynomials (1) and (2) we select $w+1-\eta_{k}^{ \pm}$that are independent.

\section{LEMMA 1. The collections}

$$
\mathscr{R}_{k}^{+}=\left\{\phi_{n, k}, 0 \leqslant n<k-1 ; \psi_{n, k}^{+}, 1 \leqslant n<k-1, n \text { odd }\right\}
$$

and

$$
\mathscr{R}_{k}^{-}=\left\{\phi_{n, k}, 0 \leqslant n<k-1 ; \psi_{n, k}^{-}, 1 \leqslant n \leqslant k-1, n \text { odd }\right\}
$$

are each composed of linearly independent polynomials.

Proof. From Proposition 1 it is clear that the $\phi_{n, k}, 0 \leqslant n<k-1$, are linearly independent. Moreover, $\phi_{n, k}(a, b, c)=-\phi_{n, k}(c, b, a)$, while for $n$ odd, $\psi_{n, k}^{ \pm}(a, b, c)$ $=+\psi_{n, k}^{ \pm}(c, b, a)$. Thus it suffices now to show that the $\psi_{n, k}^{ \pm}(a, b, c)$ in $\mathscr{R}_{k}^{ \pm}$are independent among themselves.

To establish this we introduce a change of basis for the space of spherical polynomials, namely the transformation

$$
(a, b, c) \mapsto(A, B+2 A, A+B+C) .
$$


Then

$$
\begin{aligned}
\psi_{n, k}^{ \pm}(a, b, c)= & \psi_{n, k}^{ \pm}(A, B+2 A, A+B+C) \\
= & d_{n, k}(A, B, C)-d_{n, k}(A+B+C, B+2 C, C) \\
& \mp(-1)^{k} d_{n, k}(A, B, C)-d_{n, k}(C, B+2 C, A+B+C) \\
= & d_{n, k}(A, B, C)-\sum_{j=0}^{n}\left(\begin{array}{c}
w-j \\
n-j
\end{array}\right) d_{j, k}(A, B, C) \\
& \mp(-1)^{k}\left(d_{w-n, k}(A, B, C)-\sum_{j=0}^{w-n}\left(\begin{array}{c}
w-j \\
n
\end{array}\right) d_{j, k}(A, B, C)\right),
\end{aligned}
$$

which for $1 \leqslant n \leqslant k-1, n$ odd, is a linear combination of the $d_{j, k}(A, B, C)$ with $0 \leqslant j \leqslant w-n-1$ and the coefficient of $d_{w-n-1, j}$ not equal to zero (except for $\psi_{k-1, k}^{+}, k$ even, which is zero). This triangularization of the $\psi_{n, k}^{ \pm}$in $\mathscr{R}_{k}^{ \pm}$demonstrates their linear independence.

The number of $\phi_{n, k}$ in $\mathscr{R}_{k}^{ \pm}$is $k-1$ and the number of $\psi_{n}^{ \pm}$in $\mathscr{R}_{k}^{ \pm}$is given in Table 1.

TABLE 1. The number of $\psi_{n, k}^{ \pm}$in $\mathscr{R}_{k}^{ \pm}$

\begin{tabular}{c|c|c}
\hline \multirow{2}{*}{$k$ even } & $\mathscr{R}^{+}$ & $\mathscr{R}^{-}$ \\
\cline { 2 - 3 }$k$ odd & $k / 2-1$ & $k / 2$ \\
& $(k-1) / 2$ & $(k-1) / 2$ \\
\hline
\end{tabular}

Thus in all cases we have, as asserted, $w+1-\eta_{k}$ independent relations.

We complete the proof of Theor $2 m$ by proving the following: Consider the family of discriminants $D_{t}=t^{2}+4 t, t=2,3,4, \ldots$ Belonging to $D_{t}$ is a wide equivalence class of quadratic forms associated to the reduced (in the wide sense) number

$$
[[1, t]]=1+\frac{1}{t+\frac{1}{1+\cdots}} .
$$

(For a discussion of the relationship between continued fractions and reduced quadratic forms see [12].) Since this "+"-continued fraction is of even length, the wide equivalence class consists of two distinct narrow equivalence classes, $A_{t}$ and $\theta A_{t}$, whose cycles of reduced forms are generated by the "- "-continued fractions $\mu_{0}=((3,2,2, \ldots, 2))$ and $\omega_{0}=((t+2))$, respectively.

Proposition 2. Set

$$
g_{n, k}^{ \pm}(t)=\frac{1}{2} \sum_{A_{t}}^{ \pm} d_{n, k}(a, b-2 a, a-b+c) .
$$

Then any nontrivial linear combination of elements of the set (of cardinality $\eta_{k}^{ \pm}$)

$$
\left\{g_{n, k}^{ \pm}(t), n \text { odd }, 1 \leqslant n<k-1\left(\leqslant k-1 \text { if }(-1)^{k}= \pm 1\right) ; g_{w, k}^{ \pm}(t)\right\}
$$

is zero for at most $2 k$ values of $t>2$. 
Proof. The cycle of reduced forms of $A_{t}$ is $\left\{Q_{p}=\left(a_{p}, b_{p}, c_{p}\right)\right\}_{0 \leqslant p \leqslant t-1}$ where $a_{p}=(p+1) t-p^{2}, \quad b_{p}=(2 p+3) t-2\left(p^{2}+p\right), \quad c_{p}=(p+2) t-(p+1)^{2}$. $\theta A_{t}$ contains the single reduced form $\bar{Q}=(1, t+2,1)$. (Note that $A_{t}=A_{t}^{\prime}$, so $\sum_{A_{t}}^{ \pm}=2\left(\sum_{A_{t}} \pm \sum_{\theta A_{t}}\right)$.) Taking $n$ odd, and recalling that

$$
d_{n, k}(a, b, c)=(-1)^{n} d_{n, k}(a,-b, c) \text {, }
$$

we have

(4) $\sum_{A_{t}} d_{n, k}(a, b-2 a, a-b+c)=\sum_{p=0}^{t-1} d_{n, k}\left((p+1) t-p^{2}, t-2 p,-1\right)$.

Under the transformation $p \mapsto t-p$ the right-hand side of (4) becomes

$$
\begin{aligned}
& \sum_{p=1}^{t} d_{n, k}\left((p+1) t-p^{2}, 2 p-t,-1\right)=-\sum_{p=1}^{t} d_{n, k}\left((p+1) t-p^{2}, t-2 p,-1\right) \\
& =-\sum_{p=0}^{t-1} d_{n, k}\left((p+1) t-p^{2}, t-2 p,-1\right)+d_{n, k}(t, t,-1)-d_{n, k}(t,-t,-1),
\end{aligned}
$$

from which we conclude that

$$
\begin{aligned}
\sum_{A_{t}} d_{n, k}(a, b-2 a, a-b+c) & =\frac{1}{2}\left(d_{n, k}(t, t,-1)-d_{n, k}(t,-t,-1)\right) \\
& =d_{n, k}(t, t,-1) .
\end{aligned}
$$

We also have

$$
\sum_{\theta A_{t}} d_{n, k}(a, b-2 a, a-b+c)=d_{n, k}(1, t,-t) .
$$

Thus for $n$ odd,

$$
\left(\sum_{A} \pm \sum_{\theta A}\right) d_{n, k}(a, b-2 a, a-b+c)=d_{n, k}(t, t,-1) \pm d_{n, k}(1, t,-t)=g_{n, k}^{ \pm}(t) \text {. }
$$

Writing

$$
d_{n, k}(a, b, c)=\sum_{\substack{2 \alpha+\beta=n \\
\alpha+\beta \leqslant k+1}}\left(\begin{array}{c}
k-1 \\
\beta
\end{array}\right)\left(\begin{array}{c}
k-1-\beta \\
\alpha
\end{array}\right) a^{\alpha} b^{\beta} c^{k-(1+\alpha+\beta)},
$$

we have

$$
\text { (5) } g_{n, k}^{ \pm}(t)=\sum_{\substack{\alpha+\beta=n \\
\alpha+\beta \leqslant k-1}}\left(\begin{array}{c}
k-1 \\
\beta
\end{array}\right)\left(\begin{array}{c}
k-1-\beta \\
\alpha
\end{array}\right)(-1)^{k-(1+\alpha+\beta)}\left(t^{\alpha+\beta} \pm t^{k-1-\alpha}\right) \text {. }
$$

If $n=k-1$, then $\alpha+\beta=k-1-\alpha$, and so $g_{n, k}^{ \pm}(t)=0$. Otherwise, for $n \leqslant k-$ $1, n$ odd, we claim that the right-hand side of (5) is a polynomial in $t$ of degree $\leqslant k-1$ whose $t^{(n+1) / 2}$ coefficient is nonzero but all of whose lower powers of $t$ vanish.

To see this, observe that $\alpha+\beta=n-\alpha$, and so $\alpha+\beta$ and $k-1-\alpha$ are each minimal for the largest possible choice of $\alpha$, namely for $\beta=1, \alpha=(n-1) / 2$. Then $\alpha+\beta=(n+1) / 2$, and $k-1-\alpha=k-((n+1) / 2) \geqslant(n+1) / 2$, since $n \leqslant k$ -1 . 
We now consider

$$
\begin{aligned}
g_{w, k}^{ \pm}(t) & =\sum_{p=0}^{t-1} d_{w, k}\left((p+1) t-p^{2}, t-2 p,-1\right) \pm d_{w, k}(1, t,-t) \\
& =( \pm 1)+\sum_{p=0}^{t-1}\left((p+1) t-p^{2}\right)^{k-1} .
\end{aligned}
$$

We claim that the sum in this expression is a polynomial in $t$ with no constant term, whence $g_{w, k}^{ \pm}(t)$ does have a constant term, namely \pm 1 .

$$
\sum_{p=0}^{t-1}\left((p+1) t-p^{2}\right)^{k-1}=\sum_{p=0}^{t-1}\left( \pm p^{w}+\text { higher powers of } t\right) .
$$

But

$$
\sum_{p=0}^{t-1} p^{w}=\frac{B_{w+1}(t)-B_{w+1}}{w+1}
$$

where $B_{s}(t)$ is the $s$ th Bernoulli polynomial. Since $w+1$ is odd, this is a polynomial in $t$ with no constant term, and our claim has been proved.

This triangularization of the polynomials in (3) shows them to be linearly independent.

The $g_{n, k}^{ \pm}(t)$ for $n$ odd have degree at most $k-1$, while the degree of $g_{w, k}^{ \pm}(t)$ is $2 k-1$. Thus any nontrivial linear combination of the $g_{n, k}^{ \pm}$is a polynomial in $t$ of degree at most $w+1=2 k-1$ and so can be zero for at most $2 k-1$ values of $t$. Thus for any $2 k$ values of $t \geqslant 2$, the corresponding set of the $P_{k, D, A_{t}}^{ \pm}$must span $\mathscr{W}_{k}^{ \pm}$.

To show that we can choose the $A_{t}$ to be associated to the same real quadratic field, fix a discriminant $D>0$. The class $A_{t}$ has discriminant $t^{2}+4 t$, and since $t^{2}+4 t=f^{2} D$ is equivalent to $(t+2)^{2}-f^{2} D=4$, which is just Pell's equation, we can find infinitely many values of $t$ for which $A_{t}$ has discriminant $f^{2} D$ (for different values of $f$ ).

We note the following corollary, which we shall need in the proof of a later theorem.

Corollary. The sets $\left\{\psi_{n, k}^{+}(a, b, c)\right\}, n$ odd, $1 \leqslant n<k-1$, and $\left\{\psi_{n, k}^{-}(a, b, c)\right\}$, $n$ odd, $1 \leqslant n \leqslant k-1$, are bases for the spaces of those spherical polynomials $P$ of degree $k-1$ for which $\sum_{A}^{ \pm} P=0$ for all $A \in \mathscr{A}$.

We now consider the function

$$
f_{k, D}(z)=\frac{(-1)^{(k-1) / 2} 2^{3 k-2} D^{k-1 / 2}}{\pi\left(^{2 k-2 k-1}\right)} \sum_{(a, b, c)} \frac{1}{\left(a z^{2}+b z+c\right)^{k}},
$$

where $D$ is a positive integer, $k$ an integer $\geqslant 2$, and where the summation is over all triples of integers $(a, b, c)$ with $b^{2}-4 a c=D$. This function was introduced by Zagier in [13], where it was shown to be a cusp form of weight $2 k$ on $\mathrm{SL}_{2}(\mathbf{Z})$. Then Kohnen showed in [4] that $f_{k, D}$ is the $D$ th Fourier coefficient of the holomorphic kernel function for the Shimura-Shintani correspondence between modular forms of weight $k+\frac{1}{2}$ and weight $2 k$. We shall be interested in the functions $f_{k, D, A}(z)$, 
defined in the same way as $f_{k, D}$, except that here the summation is over only those forms $(a, b, c)$ belonging to the equivalence class $A$. The periods of the functions $f_{k, D, A}^{ \pm}=: f_{k, D, A} \pm f_{k, D, A^{\prime}}$ were studied by Kohnen and Zagier in [5]. Let us here recall the basic facts about periods of modular forms.

Let $f$ be a cusp form of weight $2 k$. Then for any integer $0 \leqslant n \leqslant 2 k-2=w$, we can define the $n$th period of $f$,

$$
r_{n}(f)=\int_{0}^{i \infty} f(z) z^{n} d z
$$

The fundamental theorems on periods are the following, our exposition being essentially that in Lang [9].

THEOREM 2 (EICHLER - SHIMURA). There are certain relations among the periods of modular forms:

$$
\begin{gathered}
r_{s}+(-1)^{s} \sum_{\substack{j=0 \\
j \text { even }}}^{s}\left(\begin{array}{l}
s \\
j
\end{array}\right) r_{w-s+j}+(-1)^{s} \sum_{\substack{j=0 \\
j \equiv s \bmod 2}}^{w-s}\left(\begin{array}{c}
w-s \\
j
\end{array}\right) r_{j}=0 \\
\sum_{\substack{j=1 \\
j \text { odd }}}^{s}\left(\begin{array}{l}
s \\
j
\end{array}\right) r_{w-s+j}+\sum_{\substack{j=0 \\
j \neq s \bmod 2}}^{w-s}\left(\begin{array}{c}
w-s \\
j
\end{array}\right) r_{j}=0 .
\end{gathered}
$$

Since $r_{j}(f)$ is real if $j$ is odd, and pure imaginary if $j$ is even, these relations can be separated into two sets of relations: one involving only the even periods, the other only the odd. These relations then define two subspaces, $V^{+}$of $\mathscr{P}_{k}^{+}$and $V^{-}$of $\mathscr{P}_{k}^{-}$, where $\mathscr{P}_{k}^{+}$(resp. $\left.\mathscr{P}_{k}^{-}\right)$is the subspace of $\mathscr{P}_{k}$ consisting of polynomials even (resp. odd) in both variables.

THEOREM 3 (EICHLER - SHIMURA). Let $S_{2 k}$ be the space of cusp forms of weight $2 k$ on $\mathrm{SL}_{2}(\mathbf{Z})$. Define the mappings $r^{ \pm}$of $S_{2 k}$ into $\mathscr{P}_{k}^{ \pm}$by

$$
r^{ \pm}(f)(x, y)=\varepsilon_{ \pm} \sum_{\substack{0 \leqslant n \leqslant w \\
(-1)^{n}= \pm 1}}\left(\begin{array}{c}
w \\
n
\end{array}\right) r_{n}(f) x^{n} y^{w-n},
$$

where $\varepsilon_{+}=-i, \varepsilon_{-}=1$.

Then $r^{-}$is an isomorphism of $S_{2 k}$ and $V^{-}$, while $r^{+}$is an isomorphism of $S_{2 k}$ with a subspace of $V^{+}$of codimension 1 , not containing $x^{w}-y^{w}$.

Recall that $P_{k, D, A}(x, y)=\sum_{A} Q(x, y)^{k-1}$.

In [5] the following theorem is proved.

THEOREM 4. Let $k \geqslant 2$, and let $A$ be an $\mathrm{SL}_{2}(\mathbf{Z})$ class of quadratic forms of discriminant $D>0, D$ not a square. Then

$$
\begin{aligned}
& \frac{(-1)^{(k+1) / 2}}{2^{3 k+1}} r^{ \pm}\left(f_{k, D, A}\right) \\
& =P_{k, D, A}(x+y,-x)-P_{k, D, A}(x-y, y)+(-1)^{k} P_{k, D, \theta A}(x,-x+y) \\
& \quad-P_{k, D, \theta A}(-y, x+y) \frac{1+(-1)^{n}}{B_{2 k}} \zeta(A, 1-k)\left(x^{w}-y^{w}\right),
\end{aligned}
$$


or, equivalently, for $(-1)^{n}= \pm 1$,

$$
\begin{aligned}
& \frac{(-1)^{(k+1) / 2}}{2^{3 k-1}}\left(\begin{array}{c}
w \\
n
\end{array}\right) r_{n}\left(f_{k, D, A}^{ \pm}\right) \\
& =\sum_{\substack{(a, b, c) \in A \\
\text { reduced }}} d_{n, k}(c-b+a, 2 a-b, a)-d_{n, k}(a, b-2 a, a-b+c) \\
& \quad+(-1)^{k} \sum_{\substack{(a, b, c) \in \theta A \\
\text { reduced }}}\left(d_{n, k}(a-b+c, b-2 c, c)-d_{n, k}(c, 2 c-b, c-b+a)\right) \\
& \quad+\left(\delta_{w, n}-\delta_{0, n}\right) \frac{2 k}{B_{2 k}} \zeta(A, 1-k),
\end{aligned}
$$

where $B_{s}$ is the sth Bernoulli number, $\delta_{j, k}$ the Kronecker delta, and $\zeta(A, s)$ the Dedekind zeta function of the ideal class associated to $A$.

We can now state and prove our second result.

THEOREM 5. Let $f_{k, D, A}$ be defined as above. For $k$ an integer greater than 1 , the collection of the $f_{k, D, A}, A \in \mathscr{A}$, spans $S_{2 k}$, the space of cusp forms on $\mathrm{SL}_{2}(\mathbf{Z})$ of weight $2 k$.

Proof. By Theorems 3 and 4, if suffices to show that the polynomials $r^{+}\left(f_{k, D, A}^{+}\right)$ span the image of $r^{+}\left(S_{2 k}\right)$ as defined in Theorem 3 .

LEMMA 2. $f_{k, D, A}^{ \pm}=(-1)^{k} f_{k, D, \theta A}^{ \pm}$.

Proof. If $(a, b, c) \in A$, then $(-a,-b,-c) \in \theta A^{\prime}$. Thus $f_{k, D, A}=(-1)^{k} f_{k, D, \theta A^{\prime}}$, and the result follows at once from the definition of $f_{k, D, A}^{ \pm}$.

From Lemma 2 and Theorem 4, as well as the fact that $(a, b, c) \in A \Rightarrow(c, b, a)$ $\in A^{\prime}$, we immediately deduce

LEMMA 3. Let

$$
\begin{aligned}
S_{n, k}(a, b, c)= & -d_{n, k}(a-b+c, b-2 a, a) \\
& -d_{n, k}(c-b+a, b-2 c, c)+d_{n, k}(c, b-2 c, c-b+a) .
\end{aligned}
$$

The even periods of $f_{k, D, A}^{+}$are given for $2 \leqslant n \leqslant w-2$ by

$$
\begin{aligned}
& \frac{-(-1)^{(k+1) / 2}\left(\begin{array}{c}
w \\
n
\end{array}\right)}{2^{3 k-3}} r_{n}\left(f_{k, D, A}^{+}\right) \\
& =\left(\sum_{A}+(-1)^{k} \sum_{\theta A}\right)\left(-d_{n, k}(a-b+c, b-2 a, a)-d_{n, k}(c-b+a, b-2 c, c)\right. \\
& =:\left(\sum_{A}+(-1)^{k} \sum_{\theta A}\right) S_{n, k}(a, b, c) .
\end{aligned}
$$

Now, by the corollary to Theorem 1 and Lemma 3, there is a relation among these even periods of $f_{k, D, A}^{+}$if and only if some linear combinations of the $S_{n, k}, n$ even, equals a linear combination of the $S_{n, k}, n$ odd. Thus to prove Theorem 5 , it suffices 
to show that the dimension of the subspace of the spherical polynomials of degree $k-1$ spanned by the $S_{n, k}$ has dimension equal to the span of the $S_{n, k}, n$ odd (which is [ $(k-1) / 2])$ plus the dimension of $S_{2 k}$ (which is [k/6], unless $2 k \equiv 2(\bmod 12)$, in which case it is $[k / 6]-1$ ), plus 1 (for the zeroth period), which totals [2k/3] in all cases. We proceed now to calculate this dimension. For $0 \leqslant n \leqslant w$ define polynomials $\kappa_{n, k}(x, y)$ in $\mathscr{P}_{k}$ by

$$
\begin{aligned}
\kappa_{n, k}(x, y)= & (x-y)^{n}(-x)^{w-n}+(-x)^{n}(x-y)^{w-n} \\
& -(x-y)^{n} y^{w-n}-y^{n}(x-y)^{w-n} .
\end{aligned}
$$

LEMMA 4. $\sum_{n=0}^{w} S_{n, k}(a, b, c)=\sum_{n=0}^{w} d_{n, k}(a, b, c) \kappa_{n, k}(x, y)$.

PROOF.

$$
\begin{aligned}
& \sum_{n=0}^{w} S_{n, k}(a, b, c) x^{n} y^{w-n} \\
&=\sum_{n=0}^{w}\left(d_{n, k}(a, b-2 a, a-b+c)+d_{n, k}(c, b-2 c, c-b+a)\right. \\
&\left.\quad-d_{n, k}(a-b+c, b-2 a, a)-d_{n, k}(c-b+a, b-2 c, c)\right) x^{n} y^{w-n} \\
&=\left((a-b+c) x^{2}+(b-2 a) x y+a y^{2}\right)^{k-1} \\
& \quad+\left((c-b+a) x^{2}+(b-2 c) x y+c y^{2}\right)^{k-1} \\
& \quad-\left(a x^{2}+(b-2 a) x y+(a-b+c) y^{2}\right)^{k-1} \\
& \quad-\left(c x^{2}+(b-2 c) x y+(c-b+a) y^{2}\right)^{k-1} \\
&=\sum_{n=0}^{w}\left(d_{n, k}(a, b, c)\right)\left((x-y)^{n}(-x)^{w-n}+(-x)^{n}(x-y)^{w-n}\right. \\
&= \sum_{n=0}^{w} d_{n, k}(a, b, c) \kappa_{n, k}(x, y) .
\end{aligned}
$$

We now assert that the dimension of the span of the $\mathscr{P}_{n, k}$ in the space of spherical polynomials of degree $k-1$ is equal to the dimension of the subspace of $\mathscr{P}_{k}$ spanned by the $\kappa_{n, k}(x, y)$.

Proposition 3. Let $V$ and $A$ be vector spaces over a field, of dimension $N$, with bases $v_{1}, \ldots, v_{N}$ and $a_{1}, \ldots, a_{N}$ respectively, and suppose that

$$
\sum_{i=1}^{N} w_{i} \otimes a_{i}=\sum_{j=1}^{N} v_{j} \otimes b_{j}
$$

for some $w_{i}, 1 \leqslant j \leqslant N$, in $V$ and $b_{j}, 1 \leqslant j \leqslant N$, in $A$.

Then the dimensions of the subspace of $V$ generated by the $w_{i}$, and of the subspace of $A$ generated by the $b_{j}$ are equal. 
Proof. Write, for $1 \leqslant i \leqslant N$,

$$
w_{i}=\sum_{j=1}^{N} \alpha_{i j} v_{j} \quad \text { and } \quad b_{i}=\sum_{j=1}^{N} \beta_{i j} a_{j} .
$$

Then $\sum_{i=1}^{N} w_{i} \otimes a_{i}=\sum_{i=1}^{N} v_{i} \otimes b_{i}$ implies $\alpha_{i j}=\beta_{j i}$. Hence the matrices $\left(\alpha_{i j}\right)$ and ${ }^{t}\left(\beta_{i j}\right)$ are equal, whence $\left(\alpha_{i j}\right)$ and $\left(\beta_{i j}\right)$ have the same rank. Applying Proposition 3 to $A$, the space of spherical polynomials, $V=\mathscr{P}_{k}, a_{n}=S_{n, k}(a, b, c)$, and $v_{n}=$ $\kappa_{n, k}(x, y)$ proves the assertion.

For a matrix $\gamma=\left(\begin{array}{ll}a & b \\ c & d\end{array}\right)$ in $\operatorname{SL}_{2}(\mathbf{Z})$ we define an endomorphism $\Pi(\gamma)$ on $\mathscr{P}_{k}$ as follows: for any polynomial $P(x, y) \in \mathscr{P}_{k}$ let

$$
\Pi(\gamma) P(x, y)-\phi_{n, k}((x, y) \gamma)
$$

where

$$
(x, y) \gamma=(x, y)\left(\begin{array}{ll}
a & b \\
c & d
\end{array}\right)=(a x+c y, b x+d y) \text {. }
$$

Now let

$$
t=\left(\begin{array}{cc}
0 & -1 \\
1 & 1
\end{array}\right) \quad \text { and } \quad \sigma=\left(\begin{array}{ll}
0 & 1 \\
1 & 0
\end{array}\right) .
$$

Let $\mathscr{U}$ be the image of $\mathscr{P}_{k}$ under $\Psi=(1-\sigma)\left(t-t^{2}\right)$. Then a simple calculation shows that $\mathscr{U}$ is precisely the span of the $\kappa_{n, k}$. In calculating the dimension of $\mathscr{U}$, it makes no difference if we consider the action of $\mathrm{SL}_{2}(\mathbf{Z})$ on $\mathscr{P}_{k}(\mathbf{C})$ instead of on $\mathscr{P}_{k}(\mathbf{Q})$. The operator $\Pi(t)$ has two eigenspaces on the homogeneous polynomials of degree 1: $\mathbf{C}(x+\rho y)$ with eigenvalue $-\rho$, and $\mathbf{C}(x+\bar{\rho} y)$ with eigenvalue $-\bar{\rho}$ $\left(\rho=e^{(2 \pi i / 3)}\right)$, while $\Pi(\sigma)$ takes $(x+\rho y)$ to $\rho(x+\bar{\rho} y)$ and $(x+\bar{\rho} y)$ to $\bar{\rho}(x+\rho y)$.

Let $A=x+\rho y, B=x+\bar{\rho} y$. We have the direct sum decomposition

$$
\mathscr{P}_{w}(\mathbf{C})=\bigoplus_{j=0}^{k-1} \mathbf{C}\left(A^{j} B^{w-j} \oplus A^{w-j} B^{j}\right)
$$

into $\psi$-invariant subspaces, with

$$
\begin{aligned}
\Pi(\Psi)\left(A^{j} B^{w-j}+A^{w-j} B^{j}\right)= & \rho^{j} \bar{\rho}^{w-j}\left(1-\rho^{j} \bar{\rho}^{w-j}\right)\left(1-\rho^{j} \bar{\rho}^{w-j}\right) A^{j} B^{w-j} \\
& +\rho^{w-j} \bar{\rho}^{j}\left(1-\rho^{j} \bar{\rho}^{w-j}\right)\left(1-\rho^{w-j} \bar{\rho}^{j}\right) A^{w-j} B^{j},
\end{aligned}
$$

which is zero if and only if $\rho^{j} \vec{\rho}^{w-j}=1$. But

$$
\rho^{j} \bar{\rho}^{w-j}=\rho^{2 w-j}=1 \Leftrightarrow j \equiv 2 w \quad(\bmod 3) .
$$

Thus the dimension of $\mathscr{U}$ is

$$
\#\{j \mid 0 \leqslant j \leqslant k-1 ; 2 w-j \equiv 1 \text { or } 2(\bmod 3)\}=(2 k / 3),
$$

as was to be shown.

\section{REFERENCES}

1. Erich Hecke, Zur Theorie der Elliptischen Modulfunktionen, Math. Ann. 97 (1926), 210-242. Number 23 in Hecke, Mathematische Werke, Vandenhoeck \& Ruprecht, Göttingen, 1959.

2. , Analytische Funktionen und Algebraische Zahlen, Zweiter Teil, Abh. Math. Sem. Univ. Hamburg. 3 (1924), 13-236. Number 20 in Mathematische Werke.

3. Svetlana Katok, Modular forms associated to closed geodesics and arithmetic applications, Bull. Amer. Math. Soc. (N.S.) 11 (1984), 177-179. 
4. Winfried Kohnen, Beziehungen Zwischen Modulformen Halbganzen Gewichts und Modulformen Ganzen Gewichts, Schriften Nr. 131, Bonner Math., Bonn, 1981.

5. Winfried Kohnen and Don Zagier, Modular forms with rational periods (to appear).

6. _ Values of L-series of modular forms at the center of the critical strip, Invent. Math. 64 (1981), 175-198.

7. David Kramer, Applications of Gauss's theory of binary quadratic forms to zeta functions and modular forms, Trans. Amer. Math. Soc. (to appear).

8. Andrew Ogg, Modular forms and Dirichlet series, Benjamin, New York, 1969.

9. Serge Lang, Introduction to modular forms, Springer-Verlag, Berlin-Heidelberg-New York, 1976.

10. Carl L. Siegel, Berechnung von Zetafunktionen an ganzzahligen Stellen, Nachr. Akad. Wiss. Göttingen Math.-Phys. Kl. II 1969, 87-102.

11. Don Zagier, A Kronecker limit formula for real quadratic fields, Math. Ann. 213 (1975), 153-184.

12. __ Zetafunktionen und Quadratische Körper, Springer-Verlag, Berlin-Heidelberg-New York, 1981.

13. Modular forms associated to real quadratic fields, Invent. Math. 30 (1975), 1-46.

Department of Mathematics, Smith College, Northampton, Massachusetts 01063 